\title{
Polypharmacy is Associated with an Increased Risk of Bleeding in Elderly Patients with Venous Thromboembolism
}

\author{
Waltraud Leiss, $M D^{7}$, Marie Méan, $M D^{7}$, Andreas Limacher, PhD, MAS, MSc², Marc Righini, MD, \\ Kurt Jaeger, MD', Hans-Jürg Beer, MD ${ }^{5}$, Joseph Osterwalder, MD, MPH', Beat Frauchiger, $M D^{7}$, \\ Christian M. Matter, MD ${ }^{8}$, Nils Kucher, MD, Anne Angelillo-Scherrer, MD ${ }^{10}$, \\ Jacques Cornuz, MD, MPH ${ }^{17}$, Martin Banyai, MD ${ }^{12}$, Bernhard Lämmle, MD ${ }^{10}$, Marc Husmann, MD $^{13}$, \\ Michael Egloff, MD5 ${ }^{5}$, Markus Aschwanden, MD', Nicolas Rodondi, MD, MAS', and Drahomir \\ Aujesky, MD, MSc ${ }^{7}$
}

\begin{abstract}
'Department of General Internal Medicine, Bern University Hospital, Bern, Switzerland; ${ }^{2}$ Clinical Trials Unit Bern, Department of Clinical Research, and Institute of Social and Preventive Medicine, University of Bern, Bern, Switzerland; ${ }^{3}$ Division of Angiology and Hemostasis, Geneva University Hospital, Geneva, Switzerland; ${ }^{4}$ Division of Angiology, Basel University Hospital, Basel, Switzerland; ${ }^{5}$ Cantonal Hospital of Baden, Baden, Switzerland; ${ }^{6}$ Emergency Department, Cantonal Hospital of St. Gallen, St. Gallen, Switzerland; ${ }^{7}$ Department of Internal Medicine, Cantonal Hospital of Frauenfeld, Frauenfeld, Switzerland; ${ }^{8}$ Cardiovascular Research, Institute of Physiology, Zurich Center for Integrative Human Physiology, University of Zurich, and Division of Cardiology, Zurich University Hospital, Zurich, Switzerland; 'Division of Angiology, Bern University Hospital, Bern, Switzerland;

${ }^{10}$ Department of Hematology and Central Hematology Laboratory, Bern University Hospital, Bern, Switzerland; " Department of Ambulatory Care and Community Medicine, University of Lausanne, Lausanne, Switzerland; ${ }^{2}$ Division of Angiology, Cantonal Hospital of Lucerne, Lucerne,

Switzerland; ${ }^{13}$ Division of Angiology, Zurich University Hospital and University of Zurich, Zurich, Switzerland.
\end{abstract}

BACKGROUND: Polypharmacy, defined as the concomitant use of multiple medications, is very common in the elderly and may trigger drug-drug interactions and increase the risk of falls in patients receiving vitamin $K$ antagonists.

OBJECTIVE: To examine whether polypharmacy increases the risk of bleeding in elderly patients who receive vitamin $\mathrm{K}$ antagonists for acute venous thromboembolism (VTE).

DESIGN: We used a prospective cohort study.

PARTICIPANTS: In a multicenter Swiss cohort, we studied 830 patients aged $\geq 65$ years with VTE.

MAIN MEASURES: We defined polypharmacy as the prescription of more than four different drugs. We assessed the association between polypharmacy and the time to a first major and clinically relevant non-major bleeding, accounting for the competing risk of death. We adjusted for known bleeding risk factors (age, gender, pulmonary embolism, active cancer, arterial hypertension, cardiac disease, cerebrovascular disease, chronic liver and renal disease, diabetes mellitus, history of major bleeding, recent surgery, anemia, thrombocytopenia) and periods of vitamin $\mathrm{K}$ antagonist treatment as a time-varying covariate.

KEY RESULTS: Overall, 413 (49.8\%) patients had polypharmacy. The mean follow-up duration was 17.8 months. Patients with polypharmacy had a significantly higher incidence of major (9.0 vs. 4.1 events/100 patient-years; incidence rate ratio [IRR] 2.18, $95 \%$ confidence interval [CI] 1.32-3.68) and clinically relevant nonmajor bleeding (14.8 vs. 8.0 events/100 patient-years;

Received January 17, 2014

Revised April 4, 2014

Accepted July 25, 2014

Published online August 21, 2014
IRR 1.85, $95 \%$ CI 1.27-2.71) than patients without polypharmacy. After adjustment, polypharmacy was significantly associated with major (sub-hazard ratio [SHR] 1.83 , $95 \%$ CI 1.03-3.25) and clinically relevant nonmajor bleeding (SHR 1.60, 95 \% CI 1.06-2.42).

CONCLUSIONS: Polypharmacy is associated with an increased risk of both major and clinically relevant nonmajor bleeding in elderly patients receiving vitamin $\mathrm{K}$ antagonists for VTE.

KEY WORDS: thromboembolism; elderly; risk assessment; polypharmacy/ drugs.

J Gen Intern Med 30(1):17-24

DOI: $10.1007 / \mathrm{s} 11606-014-2993-8$

() Society of General Internal Medicine 2014

\section{INTRODUCTION}

Polypharmacy, defined as the concomitant use of multiple medications, is very common in elderly. ${ }^{1}$ Overall, between $13 \%$ and $39 \%$ of persons aged 65 years or older receive treatment with more than four drugs. ${ }^{1-3}$ Prior evidence, based essentially on patients with atrial fibrillation, suggests that polypharmacy may increase the risk of bleeding in patients treated with vitamin $\mathrm{K}$ antagonists. ${ }^{4-9}$ Several reasons could explain why polypharmacy is associated with bleeding. First, various drugs, such as amiodarone and erythromycin, alter pharmacokinetics and pharmacodynamics of vitamin K antagonists and may potentiate their anticoagulant effect. ${ }^{10}$ Second, several medication classes, such as sedatives and antihypertensive medications, may increase the risk of fall-related 
bleeding. ${ }^{11}$ Third, the combination of platelet inhibitors and oral anticoagulants may further increase the bleeding risk. ${ }^{12}$ Finally, polypharmacy could be an indicator of disease burden, which may in itself contribute to a higher risk of bleeding. ${ }^{13}$

Acute venous thromboembolism (VTE) is common in elderly persons, with an incidence between 2.8 and 4.1 cases per 1,000 person-years in persons aged $\geq 65$ years. ${ }^{14}$ Recurrent VTE is effectively prevented with vitamin $\mathrm{K}$ antagonist treatment for three months or longer. However, the risk of anticoagulation-related major bleeding is substantial, with an incidence rising up to $5 \%$ per year in patients aged 75 years and older. ${ }^{15}$ Although elderly patients with VTE are typically multimorbid and may receive multiple drug treatments, ${ }^{16}$ to our knowledge, the relationship between polypharmacy and the risk of bleeding has never been examined in this high-risk population. In a prospective, multicenter cohort of elderly patients receiving vitamin $\mathrm{K}$ antagonists for VTE, we sought to examine the association between polypharmacy and risk of bleeding, adjusting for multiple known bleeding risk factors.

\section{MATERIALS AND METHODS}

\section{Cohort Sample}

The study was conducted between September 2009 and September 2012 as part of an ongoing prospective, multicenter cohort study to assess long-term medical outcomes in 1,003 consecutive inpatients and outpatients aged $\geq 65$ years with acute, symptomatic VTE from all five Swiss university hospitals and four high-volume non-university hospitals. A full description of the cohort methods, including eligibility criteria, definition of VTE, and follow-up procedures, has been published elsewhere. ${ }^{17,18}$ Anticoagulant treatment (i.e., parenteral anticoagulant followed by vitamin $\mathrm{K}$ antagonists or parenteral anticoagulation alone) was left to the discretion of the managing hospital and primary care physicians. For this project, we excluded all patients who did not receive oral anticoagulation with vitamin $\mathrm{K}$ antagonists within 14 days of the index VTE event.

\section{Baseline Data Collection}

For all enrolled patients, trained study nurses prospectively collected information about baseline demographics (age, gender), comorbid conditions (active cancer, arterial hypertension, cardiac disease, cerebrovascular disease, chronic liver disease, chronic renal disease, diabetes mellitus, history of major bleeding, recent surgery), laboratory findings (hemoglobin, platelet count), VTE-related treatment (oral and parenteral anticoagulants, thrombolysis, insertion of an inferior vena cava filter), type and localization of index VTE event, and concomitant antiplatelet/non-steroidal anti-inflammatory drug therapy using standardized data collection forms. We assessed the risk of falls using two validated screening questions: ${ }^{19}$ )
Did you fall during the last year? If not, then 2) Did you notice any problem with gait, balance, or mobility? Patients who answered yes to at least one screening question were considered to be at high risk of falls. We also recorded all available international normalized ratio (INR) measurements at baseline and during follow-up using inpatient laboratory data and the patients' personal anticoagulation monitoring cards.

\section{Definition of Polypharmacy}

We also recorded the presence of polypharmacy at the time of study enrollment, defined as the prescription of more than four drugs, including St. John's wort, at the time of the index VTE event. ${ }^{4}$ The intake of vitamins or alternative medicine treatments was not considered.

\section{Study Outcomes}

Our primary outcome was the time to a first major bleeding during follow-up. We defined major bleeding as fatal bleeding, symptomatic bleeding at critical sites (intracranial, intraspinal, intraocular, retroperitoneal, intra-articular, pericardial, or intramuscular with compartment syndrome), or clinically overt bleeding with a reduction of haemoglobin of at least $20 \mathrm{~g} / \mathrm{L}$, or leading to transfusion of two or more units of packed red blood cells. ${ }^{20}$ The secondary outcome was the time to a clinically relevant non-major bleeding, defined as a bleeding event that required medical attention (a physician consultation or a visit at the emergency department), but that did not meet the criteria for major bleeding.

Follow-up included one telephone interview and two faceto-face evaluations during the first year of study participation, and then semi-annual contacts, alternating between face-toface evaluations (clinic visits or home visits in housebound patients) and telephone calls. During each visit/contact, study nurses interviewed patients to obtain information about the date and type of bleeding events and if the bleeding was fallrelated. If a clinical event occurred, information was complemented by reviewing medical charts and interviewing patients' primary care physicians and/or family members.

A committee of three independent blinded clinical experts reviewed and adjudicated all outcome events. Death was considered to be bleeding-related if it followed an intracranial hemorrhage or a bleeding episode leading to hemodynamic deterioration. All other deaths were classified as not bleedingrelated. ${ }^{21}$ Final classification was based on the full consensus of this committee.

\section{Statistical Analyses}

We compared baseline characteristics of patients with and without polypharmacy using Fisher's exact test for categorical variables and the non-parametric Wilcoxon rank-sum test for 
continuous variables. We calculated the overall incidence rates of major and clinically relevant non-major bleeding in patients with and without polypharmacy and compared the cumulative incidence of bleedings using the Kaplan-Meier technique and the logrank test. We also compared the percent of time spent in a given INR range $(<2.0,2.0-3.0,>3.0)$ by the unpaired $t-$ test, $^{22}$ excluding all international normalized ratio (INR) values measured during the first seven days of vitamin $\mathrm{K}$ antagonist treatment.

Because elderly multimorbid patients are at competing risk of dying from non-hemorrhagic causes before experiencing bleeding, we examined the associations between polypharmacy and the time of a first major and clinically relevant non-major bleeding using competing risk regression analysis, accounting for death as a competing event. ${ }^{23} \mathrm{We}$ accounted for non-hemorrhagic death as a competing event when analyzing major bleeding and overall death when analyzing clinically relevant non-major bleeding. The strength of the association between polypharmacy and bleeding is reflected by the sub-hazard ratio (SHR), which is the ratio of hazards associated with the cumulative incidence function in the presence of a competing risk. We adjusted for risk factors that had been previously shown to be associated with major bleeding, including age, female gender, pulmonary embolism at baseline, active cancer, arterial hypertension, cardiac disease, cerebrovascular disease, chronic liver and renal disease, diabetes mellitus, history of major bleeding, recent surgery, anemia, thrombocytopenia, and the periods of vitamin $\mathrm{K}$ antagonist treatment as a time-varying covariate. ${ }^{24-29}$ Because the use of antiplatelet/non-steroidal anti-inflammatory therapy was included in our definition of polypharmacy, we did not adjust for this variable in our primary analysis. However, given the known association between the use of antiplatelet/ non-steroidal anti-inflammatory drugs and the risk of bleeding in patients receiving vitamin $\mathrm{K}$ antagonists, ${ }^{12,30}$ the models were also adjusted for this variable in a secondary analysis. Moreover, to explore the potential effect of falls on the association between polypharmacy and anticoagulation-related

Table 1. Baseline Characteristics

\begin{tabular}{|c|c|c|c|c|}
\hline & $\begin{array}{l}\text { All } \\
(n=830)\end{array}$ & $\begin{array}{l}\text { Polypharmacy } \\
(n=413)\end{array}$ & $\begin{array}{l}\text { No polypharmacy } \\
(n=417)\end{array}$ & $\overline{p \text { value }}$ \\
\hline Characteristics $^{\mathrm{a}}$ & \multicolumn{4}{|c|}{$\mathrm{n}(\%)$ or median (range) } \\
\hline Age, years & $75(65-97)$ & $77(65-96)$ & $74(65-97)$ & $<0.001$ \\
\hline Female gender & $390(47.0)$ & $198(47.9)$ & $192(46.0)$ & 0.63 \\
\hline Overt pulmonary embolism & $599(72.2)$ & $307(74.3)$ & $292(70.0)$ & 0.19 \\
\hline Active cancer ${ }^{b}$ & $71(8.6)$ & $49(11.9)$ & $22(5.3)$ & $<0.001$ \\
\hline Arterial hypertension & $542(65.3)$ & $321(77.7)$ & $221(53.0)$ & $<0.001$ \\
\hline Cardiac disease $\mathrm{c}^{\mathrm{c}}$ & $199(24)$ & $146(35.4)$ & $53(12.7)$ & $<0.001$ \\
\hline Cerebrovascular disease $^{\mathrm{d}}$ & $73(8.8)$ & $55(13.3)$ & $18(4.3)$ & $<0.001$ \\
\hline Chronic liver disease $\mathrm{e}^{\mathrm{e}}$ & $12(1.4)$ & $6(1.5)$ & $6(1.4)$ & 1.00 \\
\hline Chronic renal disease ${ }^{\mathrm{f}}$ & $153(18.4)$ & $100(24.2)$ & $53(12.7)$ & $<0.001$ \\
\hline Diabetes mellitus & $131(15.8)$ & $97(23.5)$ & $34(8.2)$ & $<0.001$ \\
\hline History of major bleeding $\mathrm{g}$ & $75(9.0)$ & $52(12.6)$ & $23(5.5)$ & $<0.001$ \\
\hline Recent surgery & $115(13.9)$ & $72(17.4)$ & $43(10.3)$ & 0.003 \\
\hline High risk of falls ${ }^{i}$ & $377(45.4)$ & $239(57.9)$ & $138(33.1)$ & $<0.001$ \\
\hline Anemia $^{\mathrm{j}}$ & $281(33.9)$ & $185(44.8)$ & $96(23.0)$ & $<0.001$ \\
\hline Thrombocytopenia $^{\mathrm{k}}$ & $114(13.7)$ & $65(15.7)$ & $49(11.8)$ & 0.19 \\
\hline Antiplatelet/NSAID therapy ${ }^{1}$ & $330(39.8)$ & $238(57.6)$ & $92(22.1)$ & $<0.001$ \\
\hline Pre-existing VKA treatment & $44(5.3)$ & $31(7.5)$ & $13(3.1)$ & 0.005 \\
\hline \multicolumn{4}{|l|}{ Initial parenteral anticoagulation } & 0.009 \\
\hline Low-molecular-weight heparin & $386(46.5)$ & $184(44.6)$ & $202(48.4)$ & \\
\hline Dalteparin & $69(17.8)$ & $29(15.7)$ & $40(19.8)$ & \\
\hline Enoxaparin & $164(42.4)$ & $92(49.7)$ & $72(35.6)$ & \\
\hline Nadroparin & $153(39.5)$ & $63(34.05)$ & $90(44.55)$ & \\
\hline Unfractionated heparin & $278(33.5)$ & $159(38.5)$ & $119(28.5)$ & \\
\hline Fondaparinux & $143(17.2)$ & $58(14.0)$ & $85(20.4)$ & \\
\hline Danaparoid & $1(0.1)$ & $1(0.2)$ & & \\
\hline No parenteral anticoagulation & $22(2.7)$ & $11(2.7)$ & $11(2.6)$ & \\
\hline Inferior vena cava filter & $5(0.6)$ & $2(0.5)$ & $3(0.7)$ & 1.00 \\
\hline Thrombolysis & $24(2.9)$ & $5(1.2)$ & $19(4.6)$ & 0.006 \\
\hline
\end{tabular}

Abbreviations: NSAIDnon-steroidal anti-inflammatory drug; VKAvitamin K antagonist

${ }^{a}$ Values were missing for history of major bleeding $(0.1 \%)$, thrombocytopenia (7.0\%), and anemia (7.0\%)

${ }^{b}$ Solid or hematologic cancer requiring chemotherapy, radiation therapy, surgery, or palliative care during the last three months

${ }^{c}$ Systolic or diastolic heart failure, left or right heart failure, forward or backward heart failure, left ventricular ejection fraction of $<40 \%$, acute heart failure, or a myocardial infarction with or without ST elevation during the last three months, or history of coronary heart disease

${ }^{d}$ History of ischemic or hemorrhagic stroke or a transient ischemic attack

${ }^{e}$ Liver cirrhosis, chronic hepatitis, chronic liver failure, or hemochromatosis

${ }^{f}$ Diabetic or hypertensive nephropathy, chronic glomerulonephritis, chronic interstitial nephritis, myeloma-related nephropathy, or cystic kidney disease

${ }^{g}$ Bleeding that led to a hospital stay or transfusions

${ }^{h}$ Surgery requiring general or spinal anesthesia during the last three months

${ }^{i}$ Self-reported fall during the last year or any problem with gait, balance, or mobility

${ }^{j}$ Hemoglobin $<13 \mathrm{~g} / \mathrm{dL}$ for men and $<12 \mathrm{~g} / \mathrm{dL}$ for women

${ }^{k}$ Platelet count $<150,000 / \mu \mathrm{L}$

${ }^{l}$ Use of any antiplatelet therapy, such as aspirin, clopidogrel, prasugrel, aspirin/dipyridamol, or use of non-steroidal anti-inflammatory drugs 
bleeding, we additionally adjusted for the risk of falls in another secondary analysis. While missing values at baseline were generally assumed to be normal, we assumed missing values to be abnormal in a sensitivity analysis. A two-sided $p$ value $<0.05$ was considered statistically significant. All analyses were done using Stata 12 (Stata Corporation, College Station, Texas).

\section{RESULTS}

\section{Study Sample}

Of 1,003 patients enrolled in the cohort, we excluded ten patients who withdrew consent within one day from inclusion or who withdrew consent during follow-up and did not allow use of data. After the exclusion of 163 patients who did not receive vitamin $\mathrm{K}$ antagonists during the first 14 days of VTE diagnosis, our final study sample comprised 830 patients. The 163 excluded patients who did not receive vitamin $\mathrm{K}$ antagonists did not differ in age and gender, but were statistically significantly more likely to have active cancer than the 830 analyzed patients $(52.1 \%$ vs. $8.6 \%$; $p<0.001)$. All included patients were European Caucasians.

Overall, 413 patients (49.8\%) had polypharmacy. Patients with polypharmacy were significantly older and were more likely to have concomitant active cancer, arterial hypertension, cardiac disease, cerebrovascular disease, chronic renal disease, diabetes mellitus, history of major bleeding, recent surgery, high risk of falls, and anemia than patients without polypharmacy (Table 1). They were also more likely to receive antiplatelet/non-steroidal anti-inflammatory drug therapy and preexisting vitamin $\mathrm{K}$ antagonist treatment, and less likely to receive thrombolytic therapy, than patients without polypharmacy.

\section{Comparison of Bleeding}

During a mean ( \pm standard deviation) follow-up duration of $17.8( \pm 9.3)$ months following VTE, we observed 182 first bleeding episodes (75 major and 126 clinically relevant nonmajor bleedings); 87 patients died. Overall, six (8.0 \%) major bleeds were intracranial and four $(5.3 \%)$ were fatal. The overall incidences of major and clinically relevant non-major bleeding were 6.4 and 11.3 events per 100 patient-years, respectively. Patients with polypharmacy had a significantly higher incidence of major bleeding ( $9.0 \mathrm{vs.} 4.1$ events per 100 patient-years; incidence rate ratio [IRR] 2.18, $95 \%$ confidence interval $[\mathrm{CI}]$ 1.32-3.68) and clinically relevant non-major bleeding (14.8 vs. 8.0 events per 100 patient-years; IRR $1.85,95 \%$ CI 1.27-2.71) than patients without polypharmacy. As shown in Fig. 1 (Panel A and B), patients with polypharmacy had a significantly higher cumulative incidence
A

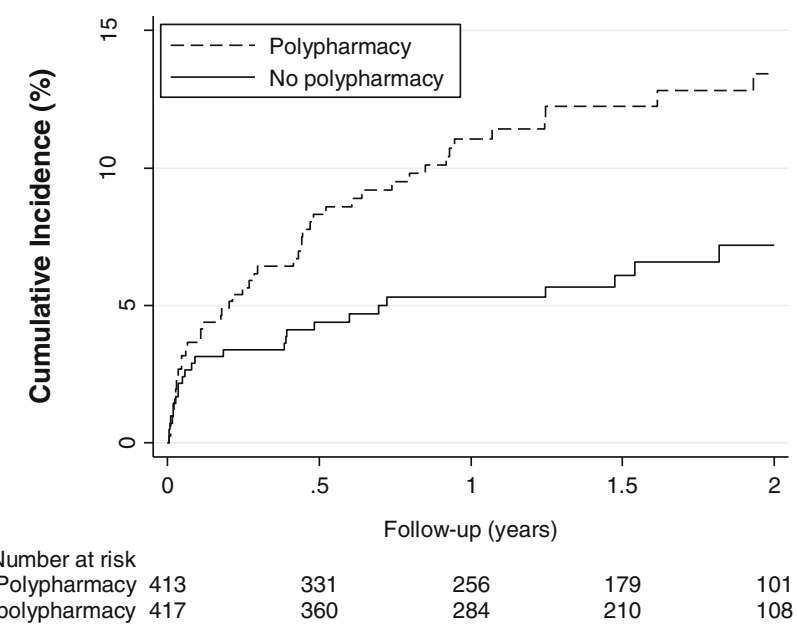

B

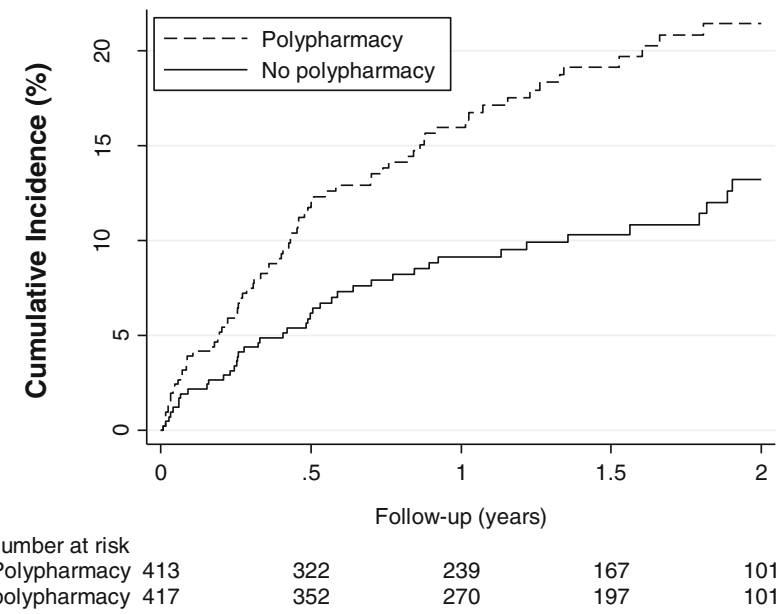

Figure 1. Panel A. Kaplan-Meier estimates for major bleeding by polypharmacy. Patients with polypharmacy had a higher 2-year cumulative incidence of major bleeding than patients without polypharmacy $(13.4 \%$ vs. $7.2 \% ; p=0.002$ by the logrank test). Panel B. Kaplan-Meier estimates for clinically relevant non-major bleeding by polypharmacy. Patients with polypharmacy had a higher 2-year cumulative incidence of clinically relevant non-major bleeding than patients without polypharmacy $(21.4 \%$ vs. $13.2 \%$; $p<0.001$ by the logrank test).

of major and clinically relevant non-major bleedings than patients without polypharmacy.

Overall, patients spent $26.0 \%, 57.4 \%$, and $16.6 \%$ of time in a subtherapeutic $(<2.0)$, therapeutic $(2.0-3.0)$, and supratherapeutic $(>3.0)$ INR range, respectively. Patients with polypharmacy spent less time in the therapeutic INR range $(54.7 \%$ vs. $60.0 \% ; p<0.001)$ and more time in a subtherapeutic INR range than patients without polypharmacy $(27.8 \%$ vs. $24.3 \% ; p=0.02$ ). Overall, patients with polypharmacy spent a non-significantly higher percentage of time in a supratherapeutic INR range than patients without polypharmacy $(17.5 \%$ vs. $15.7 \%$; $p=0.14)$.

At the time of bleeding (major and clinically relevant nonmajor), patients with polypharmacy were significantly more 
likely to have a supratherapeutic INR value $(>3.0)$ than patients without polypharmacy (49.2 \% vs. $27.9 \%$; $p=0.03)$.

Overall, 34 of 182 first bleeds (18.7\%) were fall-related. Patients with polypharmacy had a significantly higher overall incidence of a first fall-related bleeding (4.8 vs. 1.6 events per 100 patient-years; IRR 2.98, $95 \%$ CI 1.41-6.88).

\section{Association Between Polypharmacy and Bleeding}

After adjustment for previously described bleeding risk factors and periods of vitamin $\mathrm{K}$ antagonist treatment as a timevarying covariate, polypharmacy was associated with the time to a first major bleeding (adjusted SHR 1.83, $95 \%$ CI 1.033.25; Fig. 2) and the time to a first clinically relevant nonmajor bleeding (adjusted SHR 1.60, 95 \% CI 1.06-2.42; Fig. 3). Besides polypharmacy, only chronic liver disease was statistically significantly associated with major bleeding (SHR 3.53, $95 \%$ CI 1.10-11.34). After additional adjustment for antiplatelet/non-steroidal anti-inflammatory therapy, the magnitude of the association between polypharmacy and major (SHR 1.74, 95 \% CI 0.96-3.14) and clinically relevant non-major bleeding (SHR 1.43, $95 \%$ CI 0.93-2.20) decreased somewhat (Table 2).

When we further adjusted for the risk of falls, the magnitude of the association between polypharmacy and major (SHR $1.70,95 \%$ CI 0.94-3.08) and clinically relevant non-major bleeding (SHR 1.27, 95 \% CI 0.82-1.97) decreased even more (Table 2). When we assumed missing values to be abnormal in a sensitivity analysis, the results did not change markedly.

\section{DISCUSSION}

In this prospective study of elderly patients receiving vitamin $\mathrm{K}$ antagonists for acute VTE, we found that after multiple adjustments, patients with polypharmacy had a significantly higher risk of major and medically relevant non-major bleeding than patients without polypharmacy. There are several potential explanations for the observed association between polypharmacy and bleeding in our study. First, patients with polypharmacy spent more time in the subtherapeutic INR range than patients without polypharmacy, indicating that drug interactions potentiating the effect of vitamin $\mathrm{K}$ antagonists were not the primary cause of bleeding in our study. However, patients with polypharmacy

\begin{tabular}{ll}
\hline Clinical characteristic & $\begin{array}{c}\text { Adjusted sub-hazard ratio } \\
\text { (95\% confidence interval) }\end{array}$ \\
\hline Polypharmacy & $1.83(1.03$ to 3.25$)$ \\
Age (per 10 years) & $1.06(0.74$ to 1.50$)$ \\
Female gender & $1.32(0.80$ to 2.16$)$ \\
Overt pulmonary embolism & $1.12(0.66$ to 1.92$)$ \\
Active cancer & $0.75(0.32$ to 1.77$)$ \\
Arterial hypertension & $0.73(0.43$ to 1.25$)$ \\
Cardiac disease & $1.49(0.89$ to 2.50$)$ \\
Cerebrovascular disease & $0.82(0.36$ to 1.84$)$ \\
Chronic liver disease & $3.53(1.10$ to 11.34$)$ \\
Chronic renal disease & $1.52(0.87$ to 2.64$)$ \\
Diabetes mellitus & $0.88(0.47$ to 1.66$)$ \\
History of major bleeding & $1.37(0.69$ to 2.71$)$ \\
Recent surgery & $1.14(0.57$ to 2.28$)$ \\
Anemia & $1.28(0.74$ to 2.21$)$ \\
\hline & $0.96(0.49$ to 1.88$)$ \\
\hline
\end{tabular}

Figure 2. Association between polypharmacy and the time to a first major bleeding. Competing risk regression analysis, accounting for death as a competing risk. In addition, the model was also adjusted for periods of anticoagulation as a time-varying co-variate. 


\begin{tabular}{ll}
\hline Clinical characteristic & $\begin{array}{c}\text { Adjusted sub-hazard ratio } \\
\text { (95\% confidence interval) }\end{array}$ \\
\hline Polypharmacy & $1.60(1.06$ to 2.42$)$ \\
Age (per 10 years) & $1.30(0.97$ to 1.74$)$ \\
Female gender & $0.82(0.56$ to 1.19$)$ \\
Overt pulmonary embolism & $1.13(0.75$ to 1.69$)$ \\
Active cancer & $0.92(0.48$ to 1.74$)$ \\
Arterial hypertension & $0.86(0.57$ to 1.28$)$ \\
Cardiac disease & $1.07(0.70$ to 1.63$)$ \\
Cerebrovascular disease & $1.07(0.59$ to 1.95$)$ \\
Chronic liver disease & $1.88(0.53$ to 6.66$)$ \\
Chronic renal disease & $1.11(0.70$ to 1.75$)$ \\
Diabetes mellitus & $0.99(0.62$ to 1.59$)$ \\
History of major bleeding & $0.92(0.48$ to 1.75$)$ \\
Recent surgery & $0.97(0.52$ to 1.81$)$ \\
Anemia & $1.22(0.82$ to 1.83$)$ \\
\hline Thrombocytopenia & $1.12(0.68$ to 1.84$)$ \\
\hline
\end{tabular}

Figure 3. Association between polypharmacy and the time to a first clinically relevant non-major bleeding. ${ }^{\text {a }}$ Competing risk regression analysis, accounting for death as a competing risk. In addition, the model was also adjusted for periods of anticoagulation as a time-varying co-variate.

were more likely to be overanticoagulated at the time of bleeding than patients without polypharmacy (49.2 vs. $27.9 \%$ ). Overall, these findings suggest that it may be difficult to maintain constant INR levels in elderly patients with polypharmacy, with brief phases of overanticoagulation potentially resulting in bleeding. Besides drug-drug interactions, other potential reasons for more instable INR values in the elderly taking multiple drugs include the requirement of lower doses of vitamin $\mathrm{K}$ antagonists, frequent changes in medications and vitamin $\mathrm{K}$ intake with intercurrent illnesses, and suboptimal adherence. ${ }^{31}$ Second, $57.6 \%$ of patients with polypharmacy received antiplatelet/non-steroidal antiinflammatory drugs, compared to only $22.1 \%$ of patients without polypharmacy in our sample. Additional adjustment for antiplatelet/non-steroidal anti-inflammatory drugs somewhat decreased the magnitude of the association between polypharmacy and bleeding, indicating that the concomitant treatment with these drugs may be a driving factor for the increased bleeding risk in anticoagulated patients who receive multiple drug treatments.

Table 2. Adjusted Models of the Association Between Polypharmacy and the Time to a First Bleeding

\begin{tabular}{|c|c|c|c|}
\hline & \multicolumn{3}{|c|}{$\begin{array}{l}\text { Adjusted sub-hazard ratio } \\
(95 \% \text { confidence interval) }\end{array}$} \\
\hline & Base model $^{\mathbf{a}}$ & $\begin{array}{l}\text { Base model }{ }^{\mathrm{a}} \text { plus adjustment } \\
\text { for antiplatelet/ NSAID therapy }\end{array}$ & $\begin{array}{l}\text { Base model }^{\mathrm{a}} \text { plus } \\
\text { adjustment for antiplatelet/ NSAID } \\
\text { therapy and risk of falls }\end{array}$ \\
\hline \multicolumn{4}{|l|}{ Major bleeding } \\
\hline Polypharmacy & $1.83(1.03$ to 3.25$)$ & 1.74 (0.96 to 3.14$)$ & $1.70(0.94$ to 3.08$)$ \\
\hline No polypharmacy & Reference & Reference & Reference \\
\hline \multicolumn{4}{|c|}{ Clinically relevant non-major bleeding } \\
\hline Polypharmacy & $1.60(1.06$ to 2.42$)$ & $1.43(0.93$ to 2.20$)$ & $1.27(0.82$ to 1.97$)$ \\
\hline No polypharmacy & Reference & Reference & Reference \\
\hline
\end{tabular}

Abbreviation: NSAIDnon-steroidal anti-inflammatory drug

${ }^{a}$ Competing risk regression analysis, accounting for death as a competing risk. Adjustments were made for age, gender, overt pulmonary embolism, active cancer, arterial hypertension, cardiac disease, cerebrovascular disease, chronic liver disease, chronic renal disease, diabetes mellitus, history of major bleeding, recent surgery, anemia, thrombocytopenia, and periods of vitamin K antagonist treatment as a time-varying covariate 
Third, patients with polypharmacy had a higher incidence of fallrelated bleeding than patients without polypharmacy in our study. When we additionally adjusted for risk of falls, the magnitude of the association between polypharmacy and bleeding decreased even more, indicating that risk of falls may account for at least some of the relationship between polypharmacy and the risk of bleeding. Overall, although polypharmacy is known to increase the risk of falls in the elderly, mainly as a result of drug-induced sedation and orthostatic hypotension, ${ }^{11,32}$ whether an increased falls risk is associated with major bleeding remains controversial. ${ }^{8,33,34}$ Finally, a greater number of prescribed drugs may simply reflect patients' higher comorbid burden, an independent predictor of serious bleeding. ${ }^{13}$ Given that patients with polypharmacy were older and sicker in our study, despite extensive adjustment, we cannot exclude the possibility that the association between polypharmacy and bleeding is due to unmeasured potential confounders, such as overall comorbidity, acute illnesses, alcohol abuse, malnutrition, and genetic factors (e.g., CYP2C9 and VKORC1 gene mutations), rather than an effect of polypharmacy.

Our findings are difficult to compare with the results of prior studies that used differing definitions for polypharmacy, ${ }^{6,7,33}$ focused on other disease populations (e.g., patients with atrial fibrillation), ${ }^{4-9,33,35}$ and included also patients aged $<$ 65 years. ${ }^{4,5,7,8,35}$ While most studies found that the prescription of more than two to seven drugs ${ }^{4,6-8}$ was significantly associated with bleeding, others did not. ${ }^{33,35}$ In one retrospective study of 811 anticoagulated patients, of whom $74 \%$ had mechanical heart valves or atrial fibrillation, patients who bled were not taking more medications than those who did not bleed. ${ }^{35}$ However, the patients in this study were substantially younger than in our cohort and over $80 \%$ received less than two co-medications. Finally, in a prospective study of 4,093 patients aged $\geq 80$ years, of whom $74 \%$ received vitamin $\mathrm{K}$ antagonists for atrial fibrillation, the use of $\geq 3$ co-medications did not significantly increase the risk of major bleeding. ${ }^{33}$ Of note, patients were managed by professional anticoagulation clinics in this study, resulting in a very low major bleeding rate of 1.9 events per 100 patient-years only.

Our findings have clinical and research implications. Given that elderly patients with VTE who receive vitamin K antagonists and multiple drug treatments have a higher bleeding risk, such patients could potentially benefit from a more intensive anticoagulation monitoring. It seems prudent to critically review medications and to stop concomitant treatment with antiplatelet/ non-steroidal anti-inflammatory drugs and medications with an increased risk for interactions, sedation or orthostatic hypotension, if feasible. The presence of polypharmacy could also be a reason against extending vitamin $\mathrm{K}$ antagonist treatment beyond the duration of three months in such patients. Further studies are needed to examine by which mechanism(s) polypharmacy is potentially causally related to bleeding complications in patients receiving vitamin $\mathrm{K}$ antagonists. Whether new oral anticoagulants (e.g., rivaroxaban, dabigatran and apixaban) that have a lower potential for drug-drug interactions can be more safely used in elderly patients receiving multiple drug treatments should also be examined. ${ }^{36}$

Our study has potential limitations. First, given that our cohort included patients with VTE only, our results may not be generalizable to other indications for anticoagulant treatment. Second, all enrolled patients were European Caucasians. Thus, our results may not necessarily apply to other races/ ethnicities. Third, with the exception of platelet inhibitors/nonsteroidal anti-inflammatory drugs, the number and class of prescribed medications was not documented. Thus, we could not examine whether a dose-response relationship existed between the number of drugs and the bleeding risk, or whether bleeding risk was driven by specific drugs, such as antibiotics or selective serotonin or serotonin-norepinephrine reuptake inhibitors. Similarly, we had no information on the concomitant use of vitamins or alternative medicine products (except St. John's wort). Fourth, we could not assess drug adherence or whether the number of prescribed drugs changed during follow-up. Fifth, although we could adjust for the vast majority of known bleeding risk factors, we cannot exclude the possibility that the association between polypharmacy and bleeding is due to other, unmeasured confounders, such as overall comorbidity, acute illnesses, alcohol abuse, malnutrition, and genetic factors. Finally, we could only detect associations, not causality. Thus, we could not determine whether polypharmacy has a direct causal effect on bleeding, or if it is simply a marker of comorbidity.

In conclusion, elderly patients with polypharmacy who receive vitamin $\mathrm{K}$ antagonists for VTE may have a significantly increased risk of major and clinically relevant non-major bleeding. The need for antiplatelet/non-steroidal anti-inflammatory drugs and medications with interactions, sedative effect, and the potential to induce orthostatic hypotension should be critically reviewed in such patients. Further studies should explore by which mechanism polypharmacy increases the risk of bleeding, and whether less interacting new oral anticoagulants could decrease bleeding risk in patients with polypharmacy.

Acknowledgements: This work was supported by a grant of the Swiss National Science Foundation (no. 33CSCO-122659/139 470). We would like to thank all collaborators of the SWITCO65+ study.

Conflict of Interest: The authors declare that they do not have a conflict of interest.

Corresponding Author: Waltraud Leiss, MD; Department of General Internal Medicine, Bern University Hospital, Bern, Switzerland (e-mail: Waltraud.Leiss@Insel.ch).

\section{REFERENCES}

1. Kaufman DW, Kelly JP, Rosenberg L, Anderson TE, Mitchell AA. Recent patterns of medication use in the ambulatory adult population of the united states: The slone survey. JAMA. 2002;287:337-44.

2. Slabaugh SL, Maio V, Templin M, Abouzaid S. Prevalence and risk of polypharmacy among the elderly in an outpatient setting: a retrospective cohort study in the Emilia-Romagna Region, Italy. Drugs Aging. 2010;27:1019-28. 
3. Chen YF, Dewey ME, Avery AJ, the Analysis Group of the Medical Research Council Cognitive Function Ageing Study. Self-reported medication use for older people in England and Wales. J Clin Pharm Ther. 2001;26:129-40.

4. Gasse C, Hollowell J, Meier CR, Haefeli WE. Drug interactions and risk of acute bleeding leading to hospitalisation or death in patients with chronic atrial fibrillation treated with warfarin. Thromb Haemost. 2005;94:537-43.

5. The Stroke Prevention in Atrial Fibrillation Investigators. Bleeding during antithrombotic therapy in patients with atrial fibrillation. The Stroke Prevention in Atrial Fibrillation Investigators. Arch Intern Med. 1996;156:409-16.

6. Kagansky N, Knobler H, Rimon E, Ozer Z, Levy S. Safety of anticoagulation therapy in well-informed older patients. Arch Intern Med. 2004; 164:2044-50.

7. Wehinger C, Stollberger C, Langer T, Schneider B, Finsterer J. Evaluation of risk factors for stroke/embolism and of complications due to anticoagulant therapy in atrial fibrillation. Stroke. 2001;32:2246-52.

8. Donze J, Clair C, Hug B, et al. Risk of falls and major bleeds in patients on oral anticoagulation therapy. Am J Med. 2012;125:773-8.

9. Abdelhafiz AH, Wheeldon NM. Risk factors for bleeding during anticoagulation of atrial fibrillation in older and younger patients in clinical practice. Am J Geriatr Pharmacother. 2008;6:1-11.

10. Holbrook AM, Pereira JA, Labiris R, et al. Systematic overview of warfarin and its drug and food interactions. Arch Intern Med. 2005; 165: 1095-106.

11. Woolcott JC, Richardson KJ, Wiens MO, et al. Meta-analysis of the impact of 9 medication classes on falls in elderly persons. Arch Intern Med. 2009; 169: 1952-60.

12. Shireman TI, Howard PA, Kresowik TF, Ellerbeck EF. Combined anticoagulant-antiplatelet use and major bleeding events in elderly atrial fibrillation patients. Stroke. 2004;35:2362-7.

13. Fihn SD, McDonell M, Martin D, et al. Risk factors for complications of chronic anticoagulation. A multicenter study. Warfarin Optimized Outpatient Follow-up Study Group. Ann Intern Med. 1993;118:511-20.

14. Rosendaal FR, Van Hylckama VA, Doggen CJ. Venous thrombosis in the elderly. J Thromb Haemost. 2007;5:310-7.

15. Pengo V, Legnani C, Noventa F, Palareti G. Oral anticoagulant therapy in patients with nonrheumatic atrial fibrillation and risk of bleeding. A Multicenter Inception Cohort Study. Thromb Haemost. 2001;85:418-22.

16. Ageno W, Agnelli G, Imberti D, et al. Risk factors for venous thromboembolism in the elderly: results of the master registry. Blood Coagul Fibrinolysis. 2008;19:663-7.

17. Méan M, Aujesky D, Lämmle B, Gerschheimer C, Trelle S, AngelilloScherrer A. Design and establishment of a biobank in a multicenter prospective cohort study of elderly patients with venous thromboembolism (SWITCO65+). J Thromb Thrombolysis. 2013;36:484-91.

18. Méan M, Righini M, Jaeger $\mathbf{K}$, et al. The Swiss cohort of elderly patients with venous thromboembolism (SWITCO65+): rationale and methodology. J Thromb Thrombolysis. 2013;36:475-83.

19. Ganz DA, Bao Y, Shekelle PG, Rubenstein LZ. Will my patient fall? JAMA. 2007;297:77-86.

20. Schulman S, Kearon C, Subcommittee on Control of Anticoagulation of the Scientific and Standardization Committee of the International
Society on Thrombosis and Haemostasis. Definition of major bleeding in clinical investigations of antihemostatic medicinal products in non-surgical patients. J Thromb Haemost. 2005;3:692-4.

21. Jakobsson C, Jimenez D, Gomez V, Zamarro C, Méan M, Aujesky D. Validation of a clinical algorithm to identify low-risk patients with pulmonary embolism. J Thromb Haemost. 2010;8:1242-7.

22. Rosendaal FR, Cannegieter SC, van der Meer FJ, Briet E. A method to determine the optimal intensity of oral anticoagulant therapy. Thromb Haemost. 1993;69:236-9.

23. Fine JP, Gray RJ. A proportional hazards model for the subdistribution of a competing risk. J Am Stat Assoc. 1999;94:496-509.

24. Olesen JB, Lip GY, Hansen PR, et al. Bleeding risk in 'real world' patients with atrial fibrillation: comparison of two established bleeding prediction schemes in a nationwide cohort. $J$ Thromb Haemost. 2011;9:1460-7.

25. Shireman TI, Mahnken JD, Howard PA, Kresowik TF, Hou Q, Ellerbeck EF. Development of a contemporary bleeding risk model for elderly warfarin recipients. Chest. 2006;130:1390-6.

26. Beyth RJ, Quinn LM, Landefeld CS. Prospective evaluation of an index for predicting the risk of major bleeding in outpatients treated with warfarin. Am J Med. 1998;105:91-9.

27. Ruiz-Gimenez N, Suarez C, Gonzalez R, et al. Predictive variables for major bleeding events in patients presenting with documented acute venous thromboembolism. Findings from the RIETE Registry. Thromb Haemost. 2008;100:26-31

28. Gage BF, Yan Y, Milligan PE, et al. Clinical classification schemes for predicting hemorrhage: results from the National Registry of Atrial Fibrillation (NRAF). Am Heart J. 2006;151:713-9.

29. Nieuwenhuis HK, Albada J, Banga JD, Sixma JJ. Identification of risk factors for bleeding during treatment of acute venous thromboembolism with heparin or low molecular weight heparin. Blood. 1991;78:2337-43.

30. Battistella M, Mamdami MM, Juurlink DN, Rabeneck L, Laupacis A. Risk of upper gastrointestinal hemorrhage in warfarin users treated with nonselective NSAIDs or COX-2 inhibitors. Arch Intern Med. 2005;165: 18992.

31. Zarraga IG, Kron J. Oral anticoagulation in elderly adults with atrial fibrillation: integrating new options with old concepts. J Am Geriatr Soc. 2013;61:143-50.

32. Ziere G, Dieleman JP, Hofman A, Pols HA, van der Cammen TJ, Stricker BH. Polypharmacy and falls in the middle age and elderly population. Br J Clin Pharmacol. 2006;61:218-23.

33. Poli D, Antonucci E, Testa S, Tosetto A, Ageno W, Palareti G. Bleeding risk in very old patients on vitamin $\mathrm{K}$ antagonist treatment: results of a prospective collaborative study on elderly patients followed by Italian Centres for Anticoagulation. Circulation. 2011;124:824-9.

34. Kämpfen $\mathbf{P}$, Méan $\mathbf{M}$, Limacher $\mathbf{A}$, et al. Risk of falls and bleeding in elderly patients with acute venous thromboembolism. J Intern Med. 2014. doi:10.1111/joim. 12236

35. Casais P, Sanchez Luceros A, Meschengieser S, Fondevila C, Santarelli MT, Lazzari MA. Bleeding risk factors in chronic oral anticoagulation with acenocoumarol. Am J Hematol. 2000;63:192-6.

36. Garcia D, Libby E, Crowther MA. The new oral anticoagulants. Blood. 2010;115:15-20. 